\title{
Nuclear astrophysics
}

Thomas Rauscher and Friedrich-Karl Thielemann

University of Basel, Switzerland

$T^{\mathrm{n}}$ the last decades, the field of Nuclear Astrophysics has greatly 1 expanded its scope and significance. It has developed into a strongly interdisciplinary field in the overlap of astronomy, astrophysics, nuclear and particle physics. The astrophysical sites investigated include the early Universe, stable (hydrostatic) burning and evolution of stellar objects. Explosive events in single and binary systems, like novae, supernovae, X-ray bursters or environments ejecting jets of hot and dense matter are also studied. Understanding the underlying nuclear processes is also important for following galactic chemical evolution, the change of elemental abundances in a galaxy with time. The nuclear equation of state is a third important link between nuclear physics and astrophysics, in addition to energy generation and nucleosynthesis. Matter at nuclear density and beyond is encountered in neutron stars and during the formation of black holes.

Investigations in Nuclear Astrophysics involve stable as well as highly unstable nuclides. In some cases the focus is on a few key reactions, in others thousands of nuclei and tens of thousands of reactions in extended reaction networks are involved. The fact that nuclear physics is so closely related to the astrophysical results makes this interdisciplinary field exciting. Not only the details in the final abundance yields are determined by nuclear reactions but also much of the dynamics. In the following, we present a selection of astrophysical environments to illustrate the main issues.

\section{Hydrostatic burning stages}

A star trying to establish equilibrium between radiation pressure and gravitational collapse will experience a series of burning stages, permitting reactions involving nuclides with increasing charge at higher temperatures. $\mathrm{H}$ burning, the first burning stage, involves a small network of reactions in the $\mathrm{Pp}$-chains (the origin of solar neutrinos) and the CNO-cycles. Famous examples for key reactions in the second burning phase, He-burning, are the reactions ${ }^{4} \mathrm{He}(2 \alpha, \gamma){ }^{12} \mathrm{C}$ (triple-alpha reaction) and ${ }^{12} \mathrm{C}(\alpha, \gamma)^{16} \mathrm{O}$. For the triple-alpha reaction, the existence of a resonance in ${ }^{12} \mathrm{C}$ at the relevant energy for stellar He-burning temperatures $\left(T \approx 1-2 \times 10^{8} \mathrm{~K}\right)$ was predicted because vital elements like $C$ and $O$ would not exist otherwise. This prediction from the early days of nuclear astrophysics was later confirmed by a nuclear physics experiment. The reaction ${ }^{12} \mathrm{C}(\alpha, \gamma){ }^{16} \mathrm{O}$ has sometimes been termed the major problem in nuclear astrophysics. Because it determines the $\mathrm{C}$ to $\mathrm{O}$ ratio, the fate of a star in its later burning phases is strongly sensitive to the actual value of this rate. Although ${ }^{12} \mathrm{C}$ is stable and the reaction has been well studied at energies $E \geq 2.4 \mathrm{MeV}$, it has not been measured at the relevant energy of $300 \mathrm{keV}$ due to the low cross section at sub-Coulomb energy. A theoretical treatment cannot provide the necessary accuracy of better than $10 \%$ because the cross section at this energy is determined by the interference of tails of three resonances. Although the temperatures of early burning stages are of the order of several $10^{7}-10^{8}$ Kelvin, the resulting interaction energies are quite low, often below the Coulomb barrier. This leads to problems in prediction as well as measurement of charged particle reaction cross sections even for stable nuclei. Underground laboratories, such as LUNA in the Gran Sasso mountains in Italy, are making advances for reactions on light nuclei by reducing the background significantly [1].

Late burning stages, such as $\mathrm{Ne}$ - and Si-burning, exhibiting higher temperatures, allow photodisintegration as an alternative to fusion reactions. A larger number of nuclides is involved but capture and photodisintegration reactions may equilibrate, leading to a simplification of the reaction networks [1]. For instance, Si-burning ends with nuclear reactions in a complete chemical equilibrium (nuclear statistical equilibrium), minimizing the total binding energy with an abundance distribution around Fe. Thus, the sequence of burning stages is terminated. Low mass stars (such as the Sun) will not even reach Ne-burning, whereas stars with masses in the range $8 M_{\text {sol }} \leq M \leq 25 M_{\text {sol }}$ experience a collapse of the Fe-core, leading to a type II supernova explosion and the formation of a neutron star.

The creation of heavier elements has to proceed in a slightly different manner and still poses some puzzles (especially in the rprocess, see below). Due to the increased Coulomb barrier, isotopes much heavier than $\mathrm{Fe}$ can only be synthesized via reactions with neutral particles, i.e. neutrons. During core and shell He-burning specific $\alpha$-induced reactions $\left({ }^{13} \mathrm{C}(\alpha, n)^{16} \mathrm{O}\right.$, $\left.{ }^{22} \mathrm{Ne}(\alpha, \mathrm{n})^{25} \mathrm{Mg}\right)$ can liberate neutrons which then build up elements up to $\mathrm{Pb}$ and $\mathrm{Bi}$ by subsequent neutron captures on stable isotopes and $\beta$-decays, starting on existing heavy nuclei around Fe. The signature of this so-called s-process (slow neutron cap-

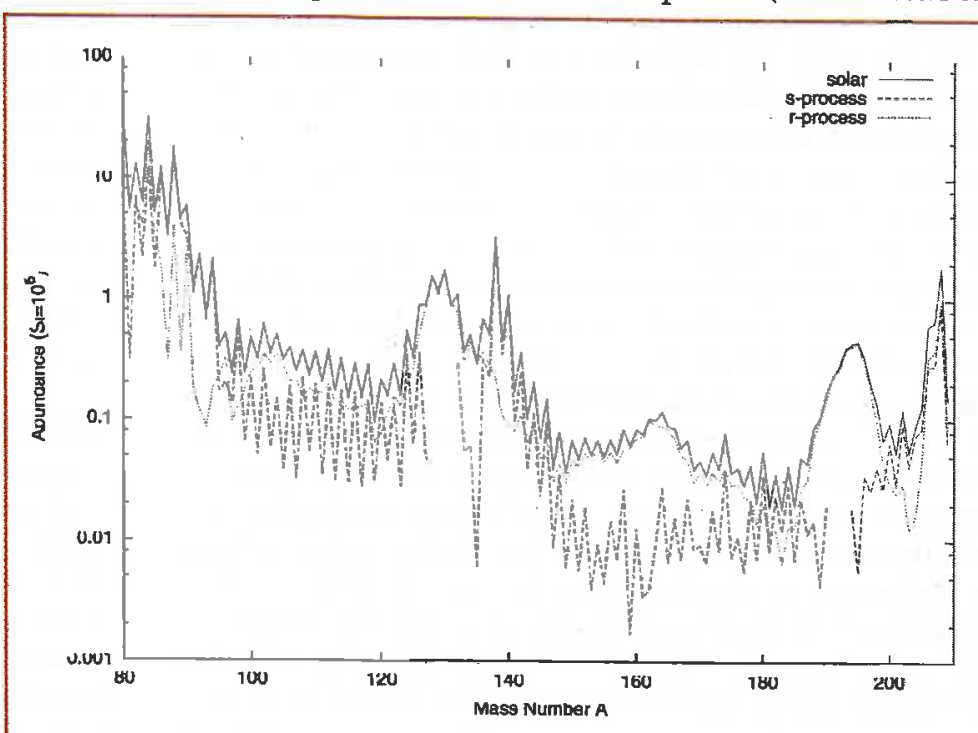

Fig. 1: Abundances for nuclides with mass number $A>80$. Solar system abundances are measured, $\mathrm{s}$ - and $\mathrm{r}$-abundances are calculated. The peaks in the solar system abundance distribution around $A=88,138,208$ are formed in the s-process, whereas the broader companion peaks shifted to slightly lower mass number are $r$-process peaks. 


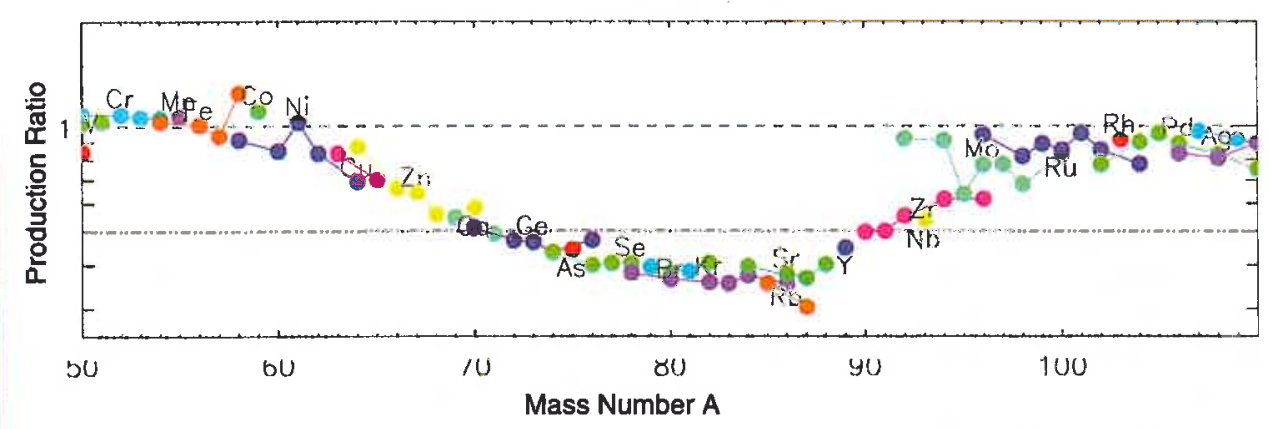

Fig. 2: Nuclides produced and ejected by a type II supernova explosion of a $25 M_{\text {sol }}$ star [7]. Shown are the ratios of two calculations with altered reaction rate sets in the mass range $50 \leq A \leq 110$. The differences are mainly due to a variation in the ${ }^{22} \mathrm{Ne}(\alpha, n)^{25} \mathrm{Mg}$ rate within current experimental uncertainties.

ture) can be found in the observed elemental abundance distribution in the Solar System (Fig. 1) where peaks are formed due to the small capture cross sections at closed neutron shells. Essential are the accurate ( $\leq 1-2 \%)$ determination of the neutron producing reactions as well as the relevant neutron capture cross sections for stable and long-lived isotopes. The effective energy is around $20-30 \mathrm{keV}$ and an important contribution can be made by classical nuclear physics experiments on stable nuclei with improved detection techniques and accuracy [2].

\section{Explosive burning}

Explosive conditions result in higher temperatures and shorter time-scales, so that often even short $\beta$-decay half-lives are longer than the reaction time-scale. This requires the inclusion of shortlived nuclei in extended networks. Two examples are the r-process and the rp-process. In an r-process (rapid neutron capture) the neutron capture times are so short that neutron-rich nuclei far off stability can be reached. An $(\mathbf{n}, \gamma)-(\gamma, \mathbf{n})$ equilibrium within an isotopic chain is established so that the time-scale is determined by the comparatively slow $\beta^{-}$-decay (even if the halflife is of the order of $\mathrm{ms}$ and the neutron separation energies are as low as 2-3 MeV). Again, the traces of such a process can be found in the solar abundances (Fig. 1). A companion peak to each s-process peak at slightly lower mass number indicates a contribution from far off stability where the closed neutron shells are encountered at a smaller mass number. Approximately half of the isotopes with $A>60$ are produced in this r-process but its site remains uncertain (type II supernovae or several sites?) [3]. The search for the actual site(s) is a major focus in the field.

Similarly, the rp-process is characterized by rapid proton captures in a proton-rich, hot environment, moving the effective synthesis path off stability toward unstable proton-rich nuclei. This process occurs in binary systems in $\mathrm{H}$-rich material, which has flown from the atmosphere of a main sequence star onto the surface of its companion neutron star. When reaching a critical mass, such an accreted layer can ignite H-burning and start a thermonuclear runaway [4]. At least one type of X-ray bursts observed in X-ray astronomy - can be connected to the rpprocess. It is yet unclear if any material can attain sufficient kinetic energy to leave the gravitational field of the neutron star during such a burst. Therefore, it remains open whether the rpprocess also contributes to nucleosynthesis.

Nuclear properties for nuclei involved in explosive processes mainly have to be predicted by theory [5]. This and therefore also the prediction of reaction rates far from stability for a large number of nuclei remains problematic. Even though microscopic models are continuously improved, phenomenological approaches are still helpful due to the sheer number of reactions to be treated. The proposed radioactive ion beam facilities in Europe, Japan and the USA will highly advance our knowledge of nuclear structure far off stability [1]. Classical nuclear physics experiments can also provide data systematics essential for the theoretical description of certain nuclear properties at energies close to the Coulomb barrier [6].

Hydrodynamic simulations have to include energy generating reaction networks influencing temperature and density profiles. Currently, many of the proposed events cannot be fully self-consistently simulated. Analyses by approximations such as parameterized reaction networks are necessary. Knowledge in nuclear physics can also be gained from analyzing results of astrophysical processes involving unstable nuclei or matter at extreme conditions. For example, the detailed analysis of the rprocess has indicated a change of neutron shell closures with distance from stable nuclei [3].

\section{Core-collapse supernovae}

The collapse of the Fe-core of a massive star leads to a type II supernova explosion, releasing a total gravitational binding energy of $2-3 \times 10^{53} \mathrm{erg}$. Neutrinos produced during the formation of the neutron star carry away this energy in the fastest fashion. The apparently most promising mechanism for obtaining explosions is based on the fact that if neutrinos deposit as few as $1 \%$ of their energy in the outer layer of the star, the material is sufficiently heated to invoke an explosion. This also leads to explosive burning and nucleosynthesis (Fig. 2) [7]. However, details of the explosion mechanism are still uncertain, as it is not yet possible to consistently simulate all aspects. Core-collapse simulations have to treat convective flows in multidimensional hydrodynamics and include a realistic nuclear equation of state as well as a correct treatment of energy transport [8]. For the latter it is necessary not only to understand radiation transport but also the interaction of neutrinos with matter. Electron captures have to be traced explicitly in the late burning phases because they are not in equilibrium. They also determine the onset of the core collapse by decreasing the stabilizing pressure of the degenerate electron gas $[1,9]$.

\section{Plasma conditions}

Of further interest is the modification of nuclear processes in stellar plasmas, which can only be studied in a very limited way in terrestrial laboratories. Target nuclei in a stellar environment are thermally excited and the reaction rate is modified in comparison to the laboratory reaction proceeding on a target in the ground state. Coulomb interactions in the plasma screen the 
potential of bare nuclei. Electron capture processes, $\beta$-decays can also be different for atoms and ions. Therefore, terrestrial halflives may be different from stellar ones.

\section{Outlook}

The potential of nuclear theory and experiment in this field has already been outlined above. Additionally, the evolution of computing power will be an important factor for future progress. Finally, new observational data on abundances and signatures of nuclear reactions will have considerable impact. Such observations can be in the form of spectra across a wide range of electromagnetic wavelengths obtained by terrestrial or satellitebased observatories from stellar surfaces, explosion remnants, and the interstellar as well as intergalactic medium. They can also be of the type of abundance determinations in specific meteoritic inclusions by means of sophisticated chemical extraction methods [10]. Such investigations become increasingly important and provide valuable additional information regarding the products of stellar nucleosynthesis. The increased inflow of such astronomical observations tremendously helps to test stellar nucleosynthesis (and with it stellar and explosive models as well as nuclear input) and, consequently, the role these events play in the evolution of galaxies.

\section{References}

[1] F. Käppeler et al., Ann. Rev. Nucl. Part. Sci. 48 (1998) 175

[2] Z.Y. Bao et al., At. Data Nucl. Data Tables 76 (2000) 70

[3] K.-L. Kratz et al, Hyperfine Interactions 129 (2000) 185; C. Freiburghaus et al, Ap. J. 516 (1999) 381

[4] H. Schatz et al, Phys. Rev. Lett. 86 (2001) 3471

[5] T. Rauscher and F.-K. Thielemann, At. Data Nucl. Data Tables 75 (2000) 1; At. Data Nudl. Data Tables 79 (2001) 1

[6] Yu.M. Gledenov et al., Phys. Rev. C 62 (2000) 042801; P. Mohr et al., Phys. Rev. C 55 (1997) 1523

[7] T. Rauscher et al., Ap. J., submitted; Nucl. Phys. A688 (2001) 193c

[8] M. Liebendörfer et al., Phys. Rev. D 63 (2001) 103004

[9] A. Heger et al., Phys. Rev. Lett. 86 (2000) 1678

[10] P. Hoppe and E. Zinner, J. Geophys. Res. - Space Phys. 105 (2000) 10371

\section{About the authors}

Thomas Rauscher received his $\mathrm{PhD}$ from the University of Technology Vienna, Austria, in 1991. Having completed a postdoc position at Harvard University, USA, he worked at the University of Mainz, Germany, as an Alexander von Humboldt fellow. Currently, he is an assistant professor at the University of Basel, Switzerland. In 1999 he received the Ludwig Boltzmann Prize of the Austrian Physical Society. His research focuses on nuclear reactions, nucleosynthesis and other topics in Nuclear Astrophysics.

Friedrich-Karl Thielemann received his $\mathrm{PhD}$ at the MaxPlanck-Institute for Astrophysics in Garching near Munich, Germany in 1980. After having held a faculty position at Harvard University, USA, he became full professor at the University of Basel, Switzerland in 1994. He is Elected Fellow of the American Physical Society and Associate Editor of Nuclear Physics A. His research interests include wide areas of nuclear physics and astrophysics. 